\title{
Numerical simulation of avalanche propagation dynamics in a rotating drum
}

\author{
Ren Han ${ }^{1}$, Jingyu Feng ${ }^{1}$, Yufeng Zhang ${ }^{1}$, Hui Yang ${ }^{1}$, Vladimir Zivkovic ${ }^{3}$, Ran $^{2} i^{2 *}$ \\ 1 School of Optical-Electrical and Computer Engineering, University of Shanghai for Science and Technology, Shanghai \\ 200093, China \\ 2 School of Medical Instrument and Food Engineering, University of Shanghai for Science and Technology, Shanghai 200093, \\ China \\ 3. School of Engineering, Newcastle University, NE1 7RU, United Kingdom
}

Corresponding author. E-mail: ran89@usst.edu.cn

\begin{abstract}
A numerical simulation based on the discrete element method (DEM) is applied to investigate the avalanche flow characteristic of particles in the slumping regime within a rotating drum. A physical experiment under the same configuration is carried out to validate the proposed model. Based on this model, the quantitative change of particles involved in avalanches on the free surface is investigated. The growth rate of avalanching particles number experiences a process of increasing, then stabilizing, and finally reducing. Investigation of the nucleation and propagation process of the avalanching reveal that any position of the free surface nucleates uniformly and the values of propagating velocities toward upward, downward, leftward, and rightward are constant. Then, it was discovered that the number of particles participating in the avalanches increases linearly with a square of time at the beginning of the avalanche, which is also proved by the simulation result. Finally, we find that the distribution of the rolling angles approximately obeys the normal distribution with the same characteristics at different times.
\end{abstract}

\section{Introduction}

Understanding and studying granular flow is important to not only prevent natural disasters such as avalanches and debris flow in nature, but also improve industrial production efficiency including granulation, drying and mixing process [1-3]. Rotating drum is an ideal tool for studying such flow patterns due to its simplicity and controllability [4-5]. In the case that a drum rotates at low speed (typically with the Froude number (Fr) between $10^{-5}$ and $10^{-3}$ ), the granular flow in the drum usually shows a series of discrete, periodic avalanches, which is so-called slumping regime operation of the rotating drum [6].

Over the years, many researchers have investigated the motion characteristics and relevant factors of the particle system in the initial stage of the avalanche. Daerr et al. observed that initial packing density and the internal structure have a strong effect on the behavior of granular flows when a pile of sand starts flowing on a rough inclined plane by a finite-sized disturbance [7]. Rabaud et al. noted that the surface velocity profile is curved and the instantaneous velocity profiles exhibit a sharp increase in the initial stage of an intermittent avalanche in a rotating drum set-up [8]. Abate et al. used speckle-visibility spectroscopy (SVS) to characterize the grain fluctuation in avalanches. The authors found that the fluctuation velocity is approximately one-tenth of the average flow velocity and these two velocities are largest at the beginning time of an avalanche [9]. Li et al. measured the avalanche dynamics in a 2D rotating drum and found that steady and unsteady states alternate. After avalanches occurring, the granular temperature in different states has different trend [10]. Recently, Yang et al. also applied SVS method finding that the particle rearrangements within the passive layer are triggered by granular avalanches occurring in the active layer [11].

These works significantly improve the insights of understanding of avalanche model. Since the behavior of granular flow is the result of the interparticle interactions, it is essential to study the avalanche process from the 
view of each particle's behavior, which is not acceptable to do by experiments at the moment. The discrete element method (DEM) keeps track of each particle and calculates the interaction between them which can reveal more flow characteristics. Therefore, some researchers take such advantages of DEM to investigate avalanches. Liu et al. observed that both the interparticle gap and the number of liquid bridges of wet particles decrease after the avalanches occur [12]. Wang et al. investigated the rich dynamic from microscopic scales in a 2D rotating drum [13]. They found that the change magnitude of stresses of all the particles increased in the initial stage of an avalanche. Yang et al. discovered that the flow velocity profile shows clear peaks as the avalanches occur [14]. These works address the characteristics of the avalanche in the initial stage by using DEM. Different from them, we investigate the flow characteristics especially from the view of tracking the motion trajectory of each particle during the whole avalanche period.

In this paper, we first introduce our numerical method and the simulation setting in section 2 . The validity of the proposed 3D simulation system is demonstrated by comparing with the experimental data under the same conditions as given in Section 3.1. Then, by tracking the trajectory of each particle, we discuss the quantitative change of particles participating in the avalanche in the drum free surface (in Section 3.2). Subsequently, we analyzed each avalanche and focus on the characteristics of nucleation sites and propagation velocities (in Section 3.3). We also investigate the probability distribution of the rolling angles and find it obeys normal distribution (in Section 3.4). Finally, a summary of the main conclusions is presented in Section 4.

\section{Simulation Method}

In this paper, a soft-sphere DEM model via Hertz-Mindlin contact forces is used here to study the slumping regime. DEM tracks individual particles and considers both interparticle and interactions with rigid boundaries [15]. We can describe the translational and rotational motion of particles by Newton's second law of motion with a force displacement correlation at the contact points of the particles. For one particle i, the governing equation for interacting with another particle $\mathrm{j}$ is

$$
\begin{gathered}
m_{i} \frac{\mathrm{d} u_{i}}{\mathrm{~d} t}=\sum_{j=1}^{n}\left(F_{c n, i j}+F_{d n, i j}+F_{c t, i j}+F_{d t, i j}\right)+G_{i} \\
I_{i} \frac{\mathrm{d} \omega_{i}}{\mathrm{~d} t}=\sum_{j=1}^{n}\left(T_{t, i j}+T_{r, i j}\right)
\end{gathered}
$$

where $\mathrm{u}_{\mathrm{i}}$ and $\omega_{\mathrm{i}}$ represent the translational velocity and angular velocity of the particle $\mathrm{i}$, respectively. The forces involved are: normal and tangential contact forces between particles $\mathrm{i}$ and particles $\mathrm{j}, \mathrm{F}_{\mathrm{cn}, \mathrm{ij}}$ and $\mathrm{F}_{\mathrm{ct}, \mathrm{j} j}$, damping forces, $F_{d n, i j}$ and $F_{d t, i j}$ and gravity, $G_{i}$. The torque of the particle $j$ acting on the particle $i$, in turn, includes 2 terms, arising from the tangential force, $\mathrm{T}_{\mathrm{t}, \mathrm{ij}}$, and the rolling friction, $\mathrm{T}_{\mathrm{r}, \mathrm{j} j}$. These forces and torques are summed over the $\mathrm{n}$ particles in contact with particle $\mathrm{i}$.

The simulation model geometry is shown in Fig.1. Periodic boundary conditions are applied along the axial direction, $x$. This treatment generates enough data for analysis while using a small number of particles (52000 particles) to minimize simulation time. Our simulations are done using commercial EDEM software () on a 32core Intel Xeon, an internal memory with a capacity of $32 \mathrm{G}$ and a GPU with a capacity of $16 \mathrm{G}$. The time step for the simulations is set to $3 \times 10^{-7} \mathrm{sec}$. The total run time is 300 seconds and the simulation time is around 100 hours. In the paper, it is assumed that the wall has the same properties as particles. The simulation conditions are summarized in Table I. 


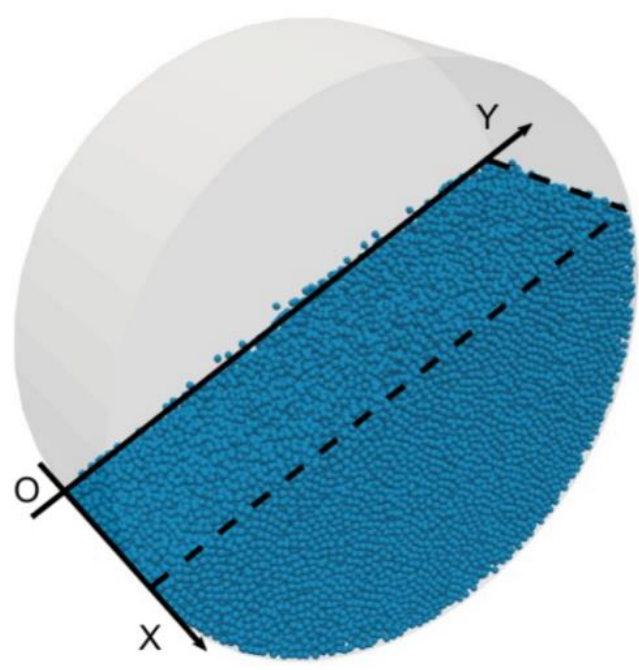

Fig.1 Simulation diagram of the three-dimensional particle system. The x-axis represents the axial direction of the drum, and the y-axis represents the radial direction.

TABLE 1. Parameters used in DEM simulations.

\begin{tabular}{ll}
\hline Parameters & Values \\
\hline Drum diameter (mm) & 140 \\
Drum length (mm) & 50 \\
Drum rotation speed (rpm) & 0.19 \\
Filling ratio f (\%) & 50 \\
Particle diameter (mm) & 2 \\
Density (kg/m ${ }^{-3}$ ) & 2650 \\
Young's modulus (Pa) & $1 \times 10^{9}$ \\
Poisson's ratio & 0.29 \\
Coef. of static friction & 0.45 \\
Coef. of rolling friction & 0.001 \\
Coef. of restitution & 0.7 \\
\hline
\end{tabular}

\section{Results and discussion}

In this section, we demonstrate the validity and additional value of the proposed simulation model on investigating the avalanche in the rotating drum. Firstly, the DEM simulation results are compared with experimentally acceptable global values like surface angle and the active surface particle velocity profile for validation purposes. Then, more detail analysis of the behavior of the particles during the avalanche, including the number of particles participating in the avalanche, the process of nucleation and propagation and the distribution of rolling angles is performed.

\subsection{Validation of the simulation}

For the validation purposes of the DEM simulations, spatial filtering velocimetry (SFV) method is adopted to obtain experimental data which is an effective method in investigating the avalanche and described in reference [17]. Experimental data and simulation results are performed under the same system configuration 
using. The granular material used here is a dry, cohesionless glass particulate material as reported in reference [6]. The rotation speed of the drum is $0.19 \mathrm{rpm}$, corresponding to a Froude number (Fr) of $8.7 \times 10^{-3}$ (Fr is defined as the ratio of centrifugal force $\omega^{2} R$ to gravity $\mathrm{g}$, ie. $\mathrm{Fr}=\omega^{2} R / \mathrm{g}$ where $\omega$ is rotation speed and $R$ is drum radius). For this rotation speed, the flows in both experiment and simulation display discrete avalanches.

Firstly, we examine the change of surface angle $\theta$ in both simulation and experiment. Fig.2 illustrates the variations of $\theta$ with time $t$ in which red line indicates simulation results and blue dots represent experimental data. It is obvious that the two curves show nearly the same change trend, which means the proposed simulation system can model the avalanche cycle effectively. The upper angles of repose are $24.7^{\circ} \pm 0.5^{\circ}$ and lower angles of repose are $21.9^{\circ} \pm 0.5^{\circ}$ by averaging the 50 consecutive avalanches in the experiment. The simulation results show the upper angles of repose are $24.5^{\circ} \pm 0.5^{\circ}$ and lower angles of repose are $21.5^{\circ} \pm 0.5^{\circ}$. The difference between the simulation and the experimental data trends are within $1^{\circ}$ even for extreme point but the averages are much closer (within $0.4^{\circ}$ ). Therefore, the simulation results are in good agreement with the experimental data.

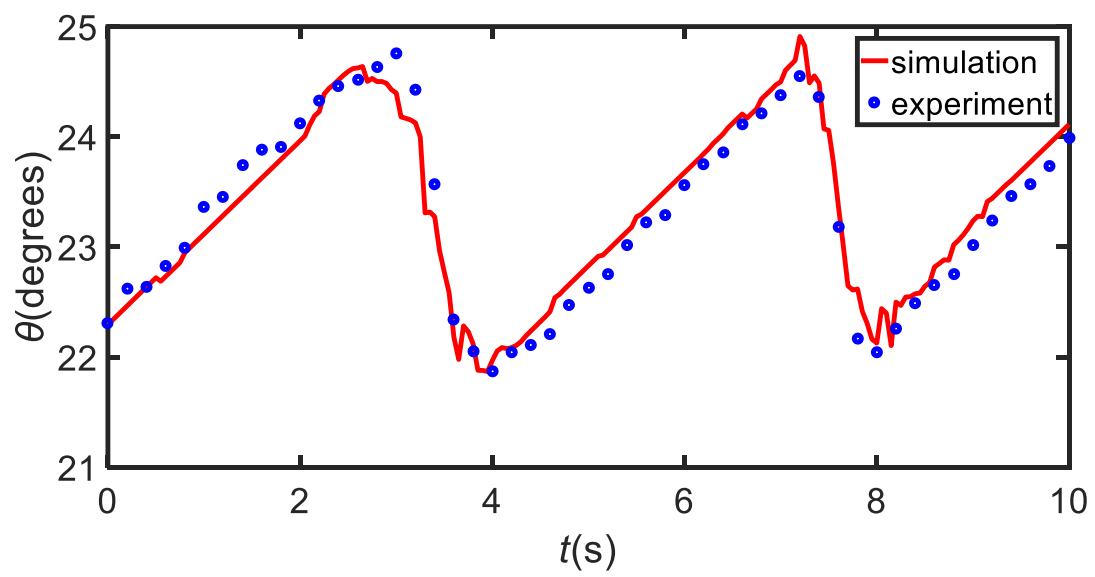

Fig. 2 Temporal variations of surface angles from simulation results vs. experimental data.

Particle velocity is another important characteristic of avalanches. Here, we evaluate the performance of the proposed simulation system on modeling the particle velocity at the drum free surface. Fig. 3 shows the distribution of particle average velocities during an avalanche along the radial direction, $y$, at midpoint axial position of the granular bed surface (averaged over 50 consecutive avalanches). As shown in Fig. 3, the simulation curve is consistent with the experiment curve. The average difference between them is $0.78 \mathrm{~mm} / \mathrm{s}$. Both of them reveal there are maximum particle velocities in the center of the bed surface. Besides, the standard deviation values of high velocity zone are greater than that of low velocity zone. Lin et al. observed similar results at higher rotating speed for drum operating in the rolling regime [18].

Based on the above described comparison using two different global properties of the drum, we validate that the proposed simulation system can accurately model the avalanche in the rotating drum. Therefore, the DEM model can provide an effective way for investigating the particle flow dynamics during avalanches. 


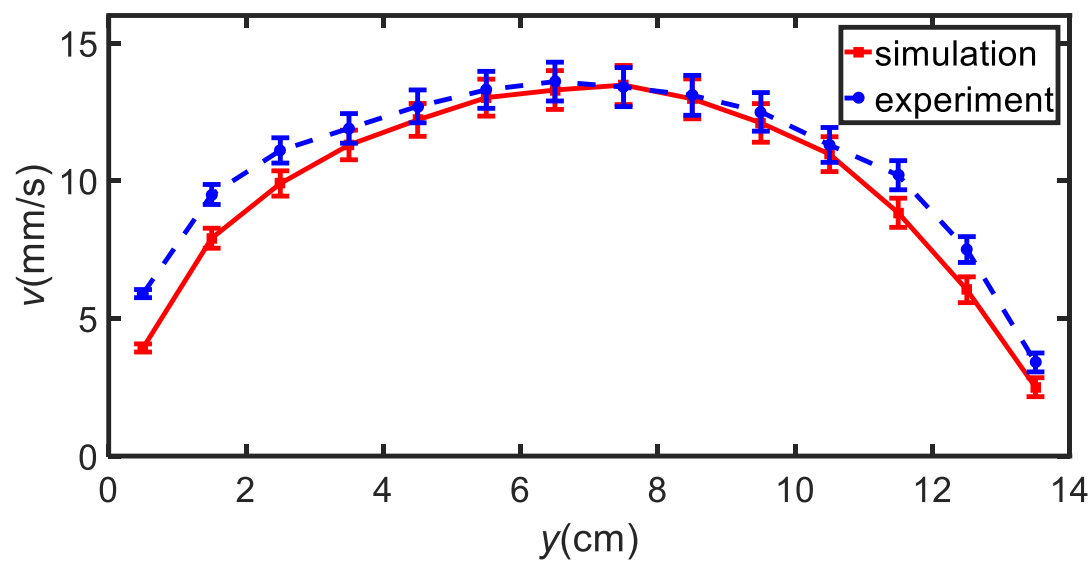

Fig.3 Particle average velocities $v$ along the radial direction at the bed surface from simulation results vs. experimental data: The error bars at each data point represent the standard deviation values in 50 measurements.

\subsection{Variation of particles number in avalanches}

Confirming the beginning and ending of an avalanche is the foundation of understanding the avalanche. However, it is still an open issue. Using particle motion to express is an intuitive method because particle motion is the direct cause of avalanche. Before the occurring of avalanche, all the particles rotate with the drum with relatively low velocities. The average velocity of particles in the surface layer is $0.5 \pm 0.03 \mathrm{~mm} / \mathrm{s}$ during this time. Once an avalanche occurs, the average velocity in the surface layer increases rapidly. Hence, the moment of the average velocity increasing to $0.6 \mathrm{~mm} / \mathrm{s}$ is considered the beginning time of the avalanche while the moment of the average velocity decreasing to $0.6 \mathrm{~mm} / \mathrm{s}$ is considered the ending time. According to this approach, it is determined that the average duration time of an avalanche, $t_{d}$, is about $1.74 \mathrm{~s}$, and the interval between successive avalanches, $t_{r}$, is about 2.52s. The interval between successive avalanches $t_{r}$ can be calculated as follows,

$$
t_{r}=\frac{\Delta \theta \pi}{180 \omega}
$$

where $\omega$ is the angular speed of the drum in rad/s. Here, the difference between the upper and lower angles $\Delta \theta$ $=2.8^{\circ}$ while rotation speed $\omega=1.14 \mathrm{rad} / \mathrm{s}$, so we get that the average interval $t_{r}=2.46 \mathrm{~s}$. This calculated result is very close to the values from simulation using velocity approach, which proves the applicability of our definition.

The number of particles involved in the avalanche is a key indicator of avalanche size. We observe that some particles stop after rolling a certain distance. Therefore, it is more reasonable to use a motion trajectory instead of speed to evaluate whether a single particle is involved in an avalanche. The position of particles at the beginning of the avalanche is labeled as the starting point. During the avalanche, particles with more than one particle diameter from its starting point are considered to participate in the avalanche. We also consider other criteria, such as one-half and one-quarter of the particle diameter displacements respectively. Simulation results reveal that different displacement criteria do not affect the variation trend of avalanche size growth (see supplementary materials). Fig.4 plots the quantitative change of particles involved in avalanches in the one particle diameter thick surface layer (averaged over 50 initial stages of avalanches). The number of particles continues to grow, which means that the avalanche size is enlarging, but the growth rate exhibits different characteristics in different periods. Based on this factor, we divide the initial stage into 3 periods, marked by T1T3 respectively. During T1 (0-0.2s), the particle number presents the trend of accelerated growth. During T2 (0.2-0.3s), the number increases with uniform acceleration (marked with a solid black line in Fig.4). During T3 $(0.3-0.5 \mathrm{~s})$, the growth rate slows down and finally reaches 0 and the number of particles increases to the maximum. 


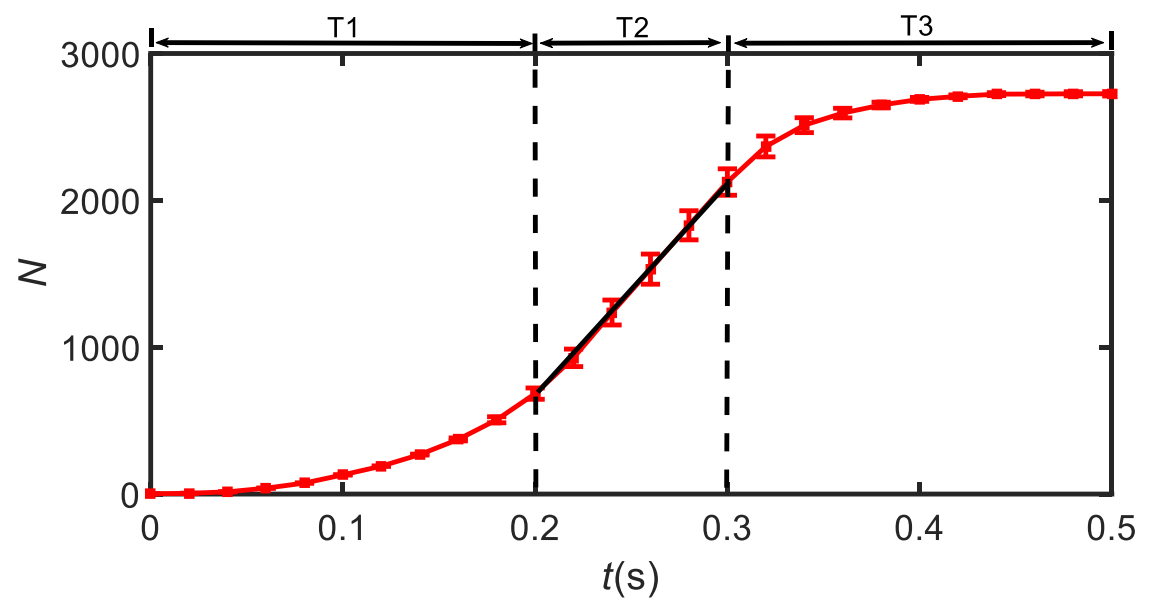

Fig.4 Number of particles involved in avalanche $N$ vs. time $t$ : The error bars for each data point represent the standard deviation of 50 measurements from independently simulated avalanche events.

\subsection{Nucleation and propagation}

In this subsection, we focus on the spatiotemporal characteristics of nucleation and propagation in the initial stage of the avalanche. In Fig.5, a series of snapshots display the nucleation and propagation on the surface of the particle bed. As can be seen from Fig.5 (a), some moving particles first appear in two different places while most particles remain stationary on the surface. Fig.5 (b) presents that in only one of the two places there exists propagation. That means not all the moving particles will lead to the next step of propagation. Such moving particles with no propagation are beyond the scope of this paper. We only studied in detail those moving particles that eventually diffuse through propagation as a nucleation site. Each nucleation site (marked in red) and propagation process is called an initial local avalanche. We also observe new moving particles constantly appearing on the surface. As shown in Fig.5 (c), nucleation sites and propagations continue to evolve. Each initial local avalanche propagates from the nucleus to the surroundings. Since the time of each nucleation is different, the size of avalanches in line with experimental results [21]. In Fig.5 (d), the scopes of initial local avalanches are large enough that their boundaries are intersecting. At that time, the avalanches cover almost the whole free surface which means most of the particles begin to roll.

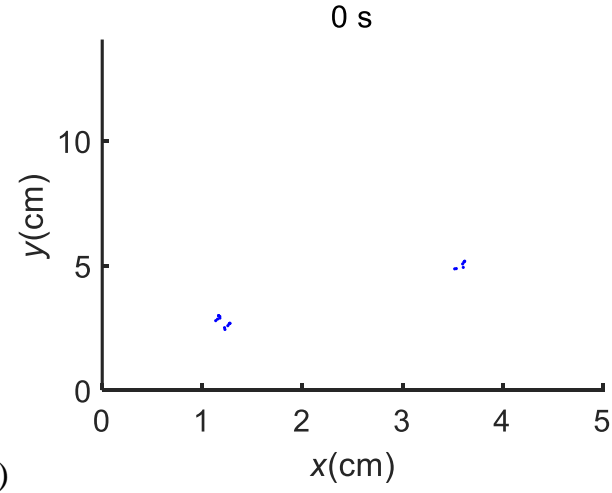

(a)

(b)

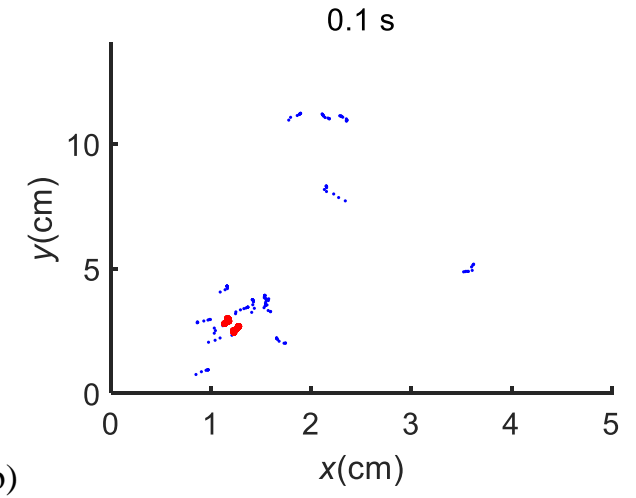



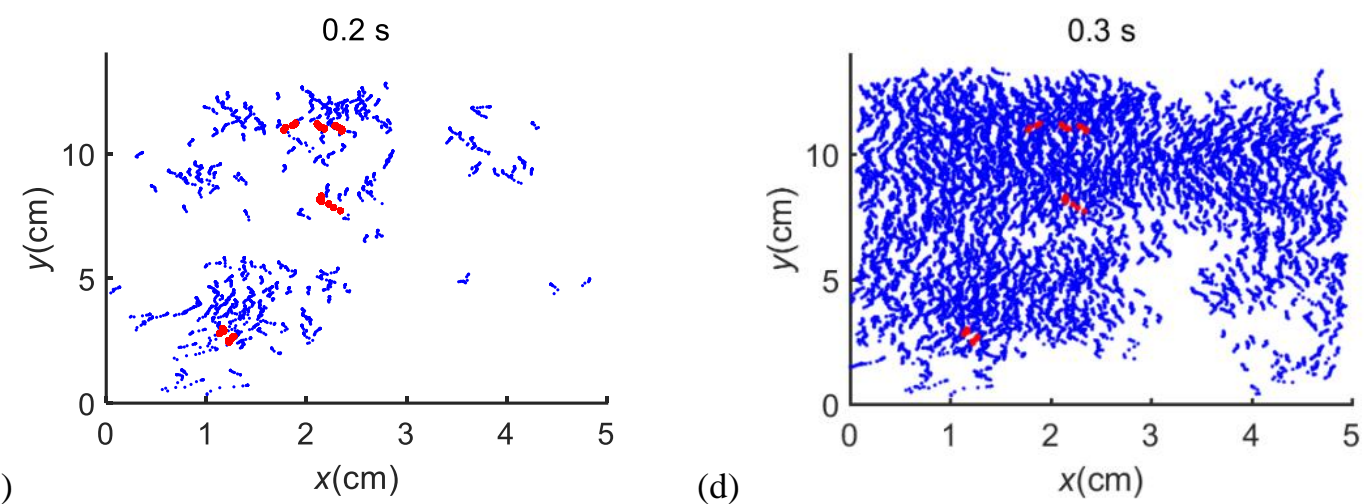

Fig.5 Sequence of snapshots showing the nucleations and the propagations in the initial stage of an avalanche. Here, the interval between each snapshot is $0.1 \mathrm{~s}$. The $\mathrm{x}$-axis and $\mathrm{y}$-axis correspond to the surface coordinate position of the particle bed in Fig.1. The nucleuses are marked in red and the trajectory of the particles is marked in blue.

The cause of nucleation and propagation is critical for the avalanche. An explanation is that with the continuous rotation of the bed surface, the most unstable particle (or several adjacent particles) is first destabilized, i.e. nucleation is started. For example, particles within the range of 3 particle diameter around the nucleus are observed to have a very small speed at the moment of the nucleation but this is quickly changed for some of them. There are many complex causes for this propagation process. The most obvious is that due to the absence of support from the particles that left the original position, the uphill adjacent particles would begin to move. Therefore, the avalanche propagates upwards. At the same time, adjacent particles lying downstream undergo shocks from the particles rolling down, triggering their motion resulting in the downward propagation. Besides, the lateral propagation can be interpreted as the rolling particles exert friction on their lateral neighbors setting them into motion. All of these effects constantly bring the original stationary particles into the avalanche.

Statistical regularity of nucleation and propagation of all the initial local avalanches is critical for studying avalanches. We investigate 500 nucleation sites and find that the first avalanching particles are uniformly distributed along both $\mathrm{x}$-axis and $\mathrm{y}$-axis (not shown here). That means any position of the free surface nucleates equally. An important question with regard to propagation is whether the propagating fronts experience an accelerated regime, or rapidly attain their constant velocities. Accordingly, we investigate the four propagating directions: $+\mathrm{X},-\mathrm{X},+\mathrm{Y}$ and $-\mathrm{Y}$ directions along $\mathrm{X}$ and $\mathrm{Y}$ axes are leftward, rightward, upward and downward directions, respectively. We define the value of the distance covered by these four fronts as the propagating distance. Fig. 6 shows the four propagating distances $S$ in the T1 phase as a function of time $t$. The experimental points have been averaged over 50 independent global avalanches events. It is important to highlight that the four sets of experimental points can be fitted to straight lines. Considering the slope of these lines reflects propagating velocities, the propagating velocity in each direction is a fixed value. As there is no external horizontal force, the propagating velocities towards left and right are almost the same. The upward velocity is about twice the downward velocity. Since the propagating velocities in these four directions are constant, we can deduce the number of particles involved in an avalanche by the following formula,

$$
N=k \times\left(V_{\text {left }}+V_{\text {right }}\right) \times\left(V_{\text {up }}+V_{\text {down }}\right) \times t^{2}
$$

where $t$ is the time after original avalanche initiation and $k$ is a constant which reflects the spatial distribution of the bed surface. In order to verify the validity of Eq. (4), we compare the calculated values with the simulation results. As shown in Fig.7, the red curve represents the simulation result of the T1 period marked in Fig.4, and the blue curve represents the corresponding calculated results from Eq. (4) $(k=64$ here) in the same period. The two curves match well in every time point, which proves that the proposed formula is correct and the number of particles involved in the avalanche increases linearly with the square of time. It is worth noting that the formula is valid to the early stage of nucleation and propagation process. Since there is no interaction between each initial local avalanche in the initial 0.2 seconds of the global avalanche initial stage, the quantitative change approximates this acceleration law. But after this initial 0.2 seconds, perhaps because each initial local avalanche 
is too large so at least some of them start to overlaps with each other, the growth rate of particle number is slowed down.

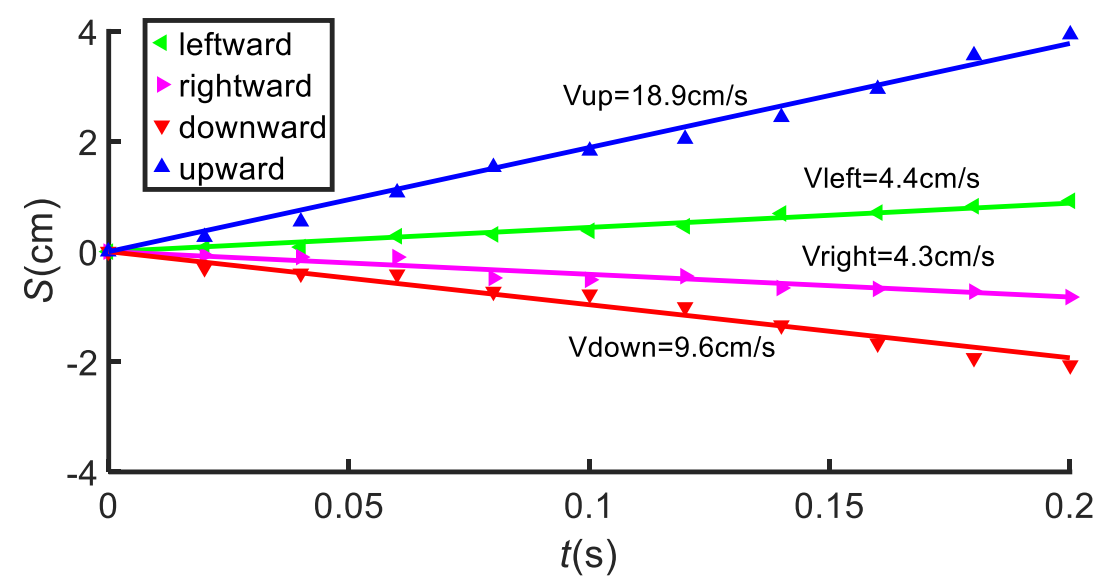

Fig.6 The four propagating distances $S$ in the T1 phase as a function of time $t$ (averaged on 50 initial local avalanches). We do not detect any transient regime in these 4 cases. The average velocity of the upward propagating front is approximately $V_{u p}=18.9 \mathrm{~cm} / \mathrm{s}$, while that of the downward, leftward and rightward propagating fronts are $V_{\text {down }}=9.6 \mathrm{~cm} / \mathrm{s}, V_{\text {left }}=4.4 \mathrm{~cm} / \mathrm{s}$ and $V_{\text {right }}=4.3 \mathrm{~cm} / \mathrm{s}$ respectively.

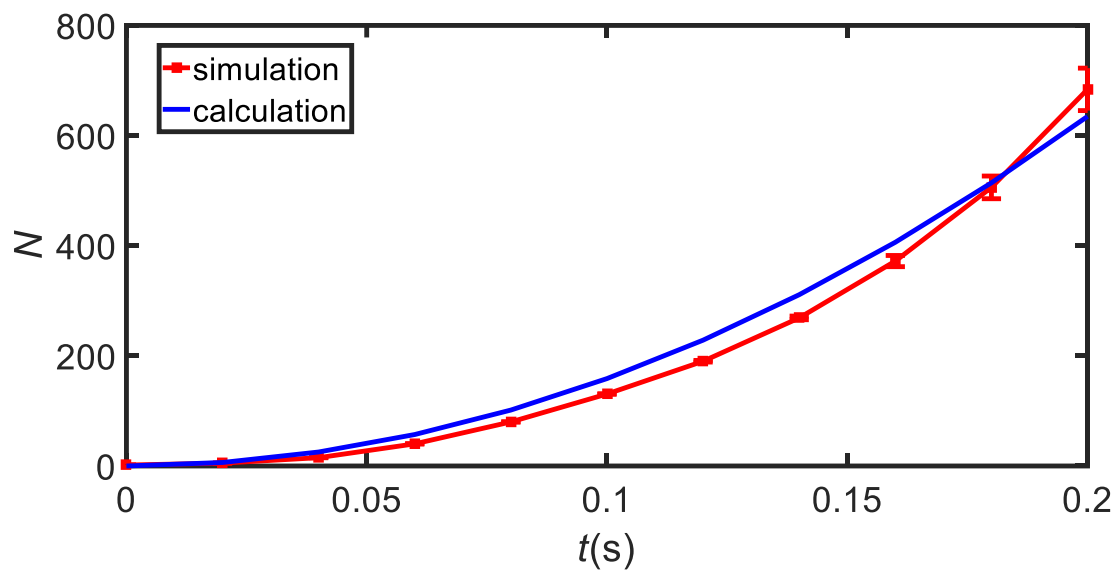

Fig.7 Number of particles involved in avalanche $N$ vs. time $t$ : The red curve represents the simulation results, and the blue curve represents the calculated results from Eq. (4).

\subsection{Distribution of rolling angles}

The rolling directions of the particles are also another interesting characteristic of avalanches. We define the angle between the line from starting point to the current position relative to y-axis as rolling angle $\theta_{r}$. The reverse direction along the $\mathrm{y}$-axis is considered to be $0^{\circ}$, the reverse direction along the $\mathrm{x}$-axis is considered to be $-90^{\circ}$, and the positive direction along the $\mathrm{x}$-axis is considered to be $90^{\circ}$. Fig. 8 reveals the distribution of the rolling angles of 5,000 particles involved in the avalanche at $0.1,0.15$ and $0.2 \mathrm{~s}$ after the avalanches nucleation and propagation start point respectively. In all three cases, the probability density distribution of the angles approximately follows the normal distribution. This indicates that this distribution law is constant over time. Secondly, $98 \%$ rolling angles are in the range of $-30^{\circ}-30^{\circ}$. We do not observe any rolling angles exceeding $60^{\circ}$ which shows that the force of the spherical particles in the $\mathrm{x}$-axis direction is relatively uniform. Furthermore, the symmetrical distribution of angles is an underlying reason for the consistency between $V_{\text {left }}$ and $V_{\text {right }}$. 


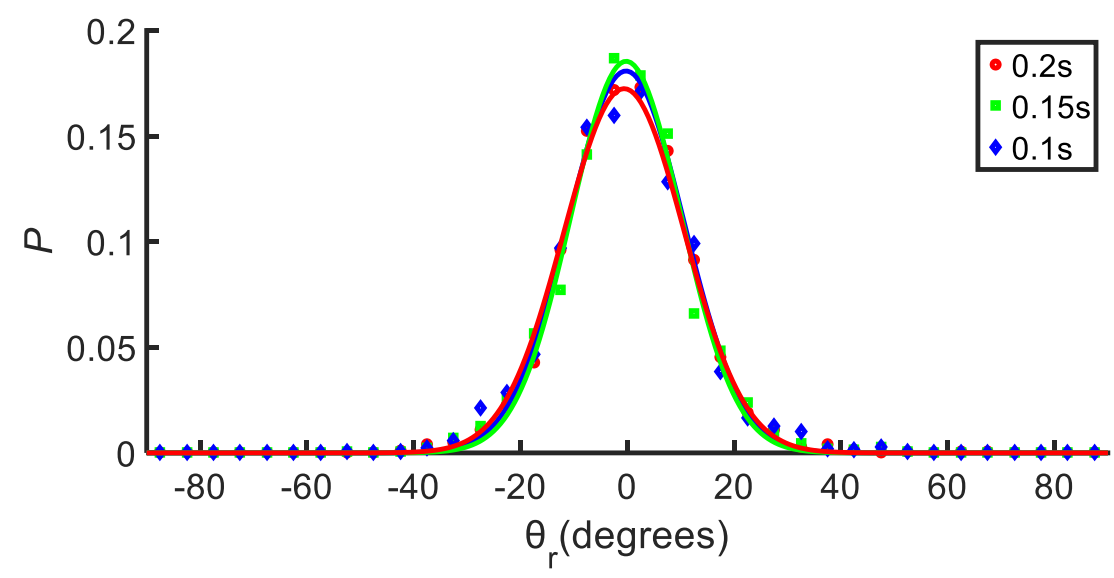

Fig.8 The probability density $P$ of the rolling angles $\theta_{r}$ of a total of 15000 particles participating in avalanches at different times is counted. The solid curve represents the best fit of the Gaussian distribution with the simulation data.

\section{Conclusion}

The granular flow behavior of the slumping flow model in a horizontal rotating drum is studied using the DEM model. The DEM simulations are validated by comparing the simulation results with the experimental data such as the drum surface angle and the particle surface velocities. Analysis of the avalanche nucleation and propagation process indicates there are three distinct temporal periods. The analysis of the trajectory of each particle shows that the growth rate of avalanche size is different in each time period: It accelerates during the T1 period, grows in uniform acceleration during the T2 period, and finally slows down and reaches 0 during the T3 period. Our results suggest that each initial local avalanche of the free surface undergoes a process of nucleation and propagation. The nucleation sites are evenly distributed and the propagating velocities are constant, with the largest velocity in the upward direction, which is approximately double the magnitude of downward propagation velocity. The propagation velocities in the radial direction are even smaller with no difference between left and right directions. On the account of these results, we derive the number of particles participating in avalanches increases linearly with the square of time which accords with the change rule mentioned in the T1 period. We also identify the probability density distribution of the rolling angles approximately obeys the same normal distribution at different times being completely symmetrical in the radial direction. The maximum probability for the rolling angle is for the axial direction $\left(0^{\circ}\right.$ angle) while $98 \%$ of rolling angles are in the range of $-30^{\circ} \sim 30^{\circ}$. Overall, all of these results indicate that the axial propagation is dominant over the radial propagation, although the radial propagation cannot be neglected.

Acknowledgment

This study has been supported by the National Natural Science Foundation of China $(91634202,11902190)$.

\section{References}

[1] H. Yang, B.F. Zhang, R. Li, G. Zheng, V. Zivkovic, Particle dynamics in avalanche flow of irregular sand particles in the slumping regime of a rotating drum, Powder Technology, 311 (2017) 439-448.

[2] E. Marteau, J.E. Andrade, A model for decoding the life cycle of granular avalanches in a rotating drum, Acta Geotechnica, 13 (2017) 549-555.

[3] J. Rajchenbach, Development of grain avalanches, Phys Rev Lett, 89 (2002) 074301. 
[4] D.A. Santos, C.R. Duarte, M.A.S. Barrozo, Segregation phenomenon in a rotary drum: Experimental study and CFD simulation, Powder Technology, 294 (2016) 1-10.

[5] N. Sepúlveda, G. Krstulovic, S. Rica, Scaling laws in granular continuous avalanches in a rotating drum, Physica A: Statistical Mechanics and its Applications, 356 (2005) 178-183.

[6] H. Yang, R. Li, P. Kong, Q.C. Sun, M.J. Biggs, V. Zivkovic, Avalanche dynamics of granular materials under the slumping regime in a rotating drum as revealed by speckle visibility spectroscopy, Phys Rev E , 91 (2015) 042206.

[7] Daerr, A., \& Douady, S, Two types of avalanche behaviour in granular media, Nature, 399 (1999) 241243.

[8] S.C. du Pont, R. Fischer, P. Gondret, B. Perrin, M. Rabaud, Instantaneous velocity profiles during granular avalanches, Phys Rev Lett, 94 (2005) 048003.

[9] A.R. Abate, H. Katsuragi, D.J. Durian, Avalanche statistics and time-resolved grain dynamics for a driven heap, Phys Rev E Stat Nonlin Soft Matter Phys, 76 (2007) 061301.

[10] R. Li, H. Yang, G. Zheng, Q.C. Sun, Granular avalanches in slumping regime in a 2D rotating drum, Powder Technology, 326 (2018) 322-326.

[11] R. Li, H. Yang, G. Zheng, B.F. Zhang, M.L. Fei, Q.C. Sun, Double speckle-visibility spectroscopy for the dynamics of a passive layer in a rotating drum, Powder Technology, 295 (2016) 167-174.

[12] P.Y. Liu, R.Y. Yang, A.B. Yu, Dynamics of wet particles in rotating drums: Effect of liquid surface tension, Physics of Fluids, 23 (2011).

[13] Z. Wang, J. Zhang, Fluctuations of particle motion in granular avalanches - from the microscopic to the macroscopic scales, Soft Matter, 11 (2015) 5408-5416.

[14] R.Y. Yang, A.B. Yu, L. McElroy, J. Bao, Numerical simulation of particle dynamics in different flow regimes in a rotating drum, Powder Technology, 188 (2008) 170-177.

[15] H. Wei, Y. Zhao, J. Zhang, H. Saxén, Y. Yu, LIGGGHTS and EDEM application on charging system of ironmaking blast furnace, Advanced Powder Technology, 28 (2017) 2482-2487.

[16] P. Tegzes, T. Vicsek, P. Schiffer, Avalanche dynamics in wet granular materials, Phys Rev Lett, 89 (2002) 094301.

[17] J.M. Gong, H. Yang, S.H. Lin, R. Li, V. Zivkovic, Spatial filtering velocimetry for surface velocity measurement of granular flow, Powder Technology, 324 (2018) 76-84.

[18] S.H. Lin, H. Yang, R. Li, G. Zheng, V. Zivkovic, Velocities of irregular particles in a continuously avalanching surface flow within a rotating drum, Powder Technology, 338 (2018) 376-382.

[19] E. Marteau, J.E. Andrade, A model for decoding the life cycle of granular avalanches in a rotating drum, Acta Geotechnica, 13 (2017) 549-555.

[20] H. Yang, R. Li, P. Kong, Q.C. Sun, M.J. Biggs, V. Zivkovic, Avalanche dynamics of granular materials under the slumping regime in a rotating drum as revealed by speckle visibility spectroscopy, Phys Rev E Stat Nonlin Soft Matter Phys, 91 (2015) 042206.

[21]H. Mou, H. Yang, R. Li, G. H. Zhang, Q. C. Sun, P. Kong. Particle dynamics of the passive layer within a granular drum using wavelet analysis. Powder Technology, 2019(344): 1-9

[22]J. Song, H. Yang, R. Li, Q. Chen, Y. J. Zhang, Y. J. Wang, P. Kong, Improved PTV measurement based on Voronoi matching used in hopper flow, Powder Technology, 2019, 355:172-182

[23]Y. J. Zhang, H.Yang, R.Li, Q.Chen, Q. C. Sun, P. Kong. Accumulation phenomenon in continuous flow of irregular particles in rotating drum, Powder Technology, 2019, 355: 333-339

[24] H. Yang, Y. H. Zhu, R. Li, Q. C. Sun. Kinetic granular temperature and its measurement using speckle visibility spectroscopy, Particuology, 2019 (in press)

[25] P. Bak, C. Tang, K. Wiesenfeld, Self-organized criticality: An explanation of the 1/f noise, Phys Rev Lett, 59 (1987) 381-384.

\section{Supplementary Material}

We investigate whether different avalanche criteria can affect the quantitative variation trend of particles participating in the avalanche. A new criterion is adopted to analyze the simulations in Section 3.2 that particles 
with more than one-half particle diameter from its starting point are considered to participate in the avalanche. Fig.9 plots the quantitative change of particles involved in avalanches in one particle diameter thick surface layer (averaged over 50 initial stages of avalanches) under such criterion. It can be seen clearly that the growth rate of particle number also experiences a process of increasing, then stabilizing, and finally reducing, which is consistent with Fig.4. Therefore, different displacement criteria do not affect the variation trend of avalanching particles number.

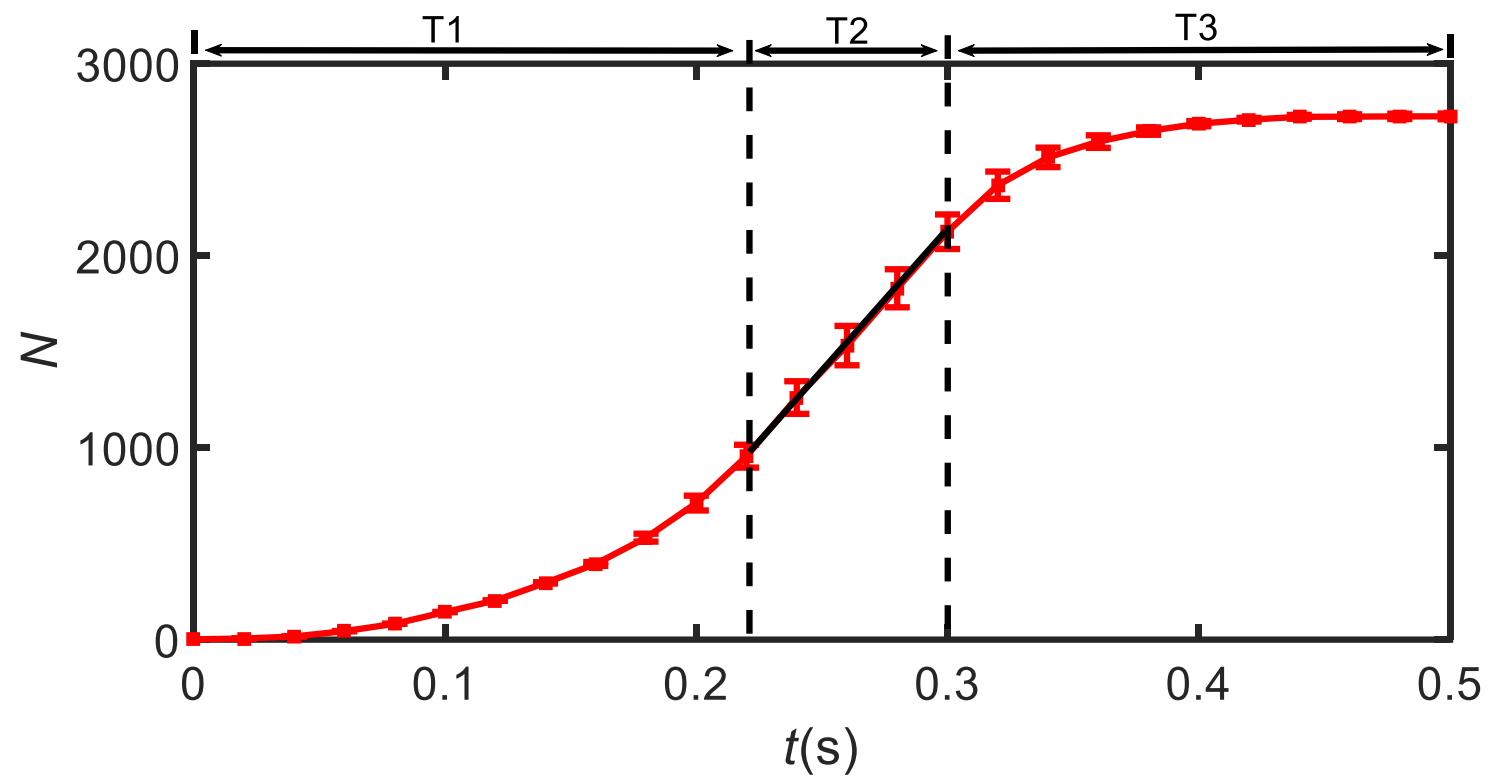

Fig.9 Number of particles involved in avalanche $N$ vs. time $t$ : The error bars for each data point represent the standard deviation of 50 measurements from independent simulated avalanche events. Particles with more than one-half particle diameter from its starting point are considered to participate in the avalanche. 